\title{
3-D Twisted Flux-Tube in a Linear Force-Free Equilibrium
}

\author{
G. Aulanier and P. Démoulin \\ Observatoire de Paris, Section de Meudon, DASOP, URA 2080 (CNRS), \\ 92195 Meudon Cedex, France
}

\begin{abstract}
It is now commonly accepted that prominence plasma is supported in magnetic dips, in particular in twisted flux tubes. But present two-dimensional models are unable to explain the observed presence and structure of prominence feet. This requires three-dimensional models. We modelled the field using linear force-free field equations. Combining a small number of harmonics, and using observational constraints, we have found the area in the parameter space where prominences are likely to be present. Then, adding 3-D harmonics, we show that feet appear periodically underneath the prominence body. For great helicity, the parameter space is mostly fulfilled by configurations which have feet alternating between both sides of the prominence axis, as observed. The lateral feet are formed by dips in the vicinity of small magnetic polarities, that are located in a low field corridor where the prominence stands. The modelled configurations show in a natural fashion many observational aspects of prominences, such as the vector magnetic field measurements in prominences and the chirality patterns.
\end{abstract}

\section{Description of the Model}

Prominences are formed of plasma sufficiently ionised that we can consider it frozen into the magnetic field. Furthermore, their long-time existence and the slow motions of the plasma inside them allow us to consider their global structure as quasi-static, to a first approximation. The plasma $\beta$ is typically of the order of 0.01 to 0.1 , so the field can almost be considered as force-free. We choose below a linear force-free field (lfff) because it provides a large class of 3-D equilibrium states permitting us to understand the presence of feet (Démoulin et al. 1989, Aulanier and Démoulin 1998). Then the lfff can be described analytically by the combination of periodic harmonics, that are solutions of:

$$
\triangle \vec{B}+\alpha^{2} \vec{B}=0 .
$$

The main criterion for a magnetic configuration that can support a prominence is the existence of dips in the field lines (Démoulin 1998, these proceedings, and references therein). In the following we will suppose that cold material is present in such dips while we neglect its effect on the magnetic field. 


\section{Filaments in OF Twisted Flux Tubes}

From a preliminary $2 \frac{1}{2}$-D study of the location of prominence-like configurations in the parameter space, it appears that among the various topologies, only the $\mathrm{OF}$ and $\mathrm{OBP}$ are satisfying (see Figure 1). The first letter of the terminology refers to the top of the prominence, and the following one(s) to its bottom. $\mathrm{O}$ is given for an O-point (i.e., a twisted flux tube), $\mathrm{F}$ for a flat field line, and BP for a bald patch. The signature of a bald patch is the presence of field lines whose dip is tangent to the photosphere (Titov et al. 1993). Hence, the underlying feet of the prominence are expected to have such a configuration in 3-D.
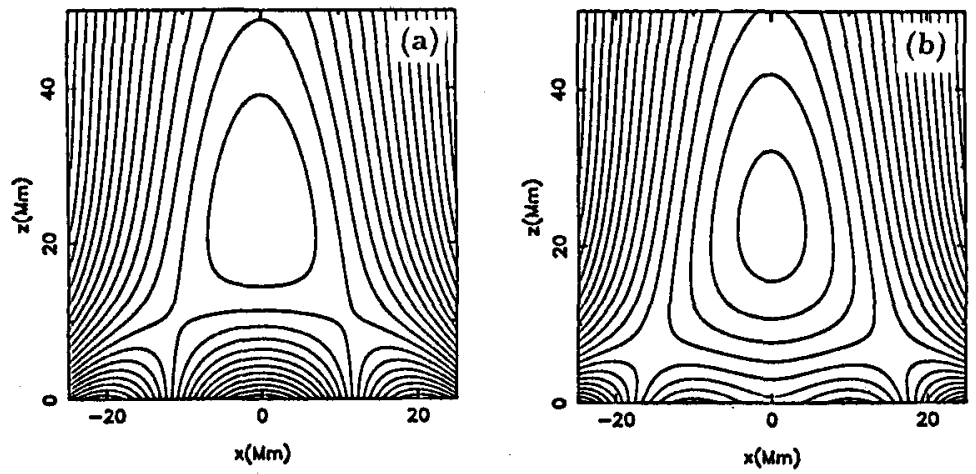

Figure 1. Topologies for the field in $2 \frac{1}{2}$-D satisfying the conditions for a prominence. The configuration is OF in (a) and OBP in (b).

\section{Observables for a 3-D Prominence}

For a given set of parameters, one can find a configuration which is satisfying for many observed aspects of prominences. The photospheric field has a quasi-
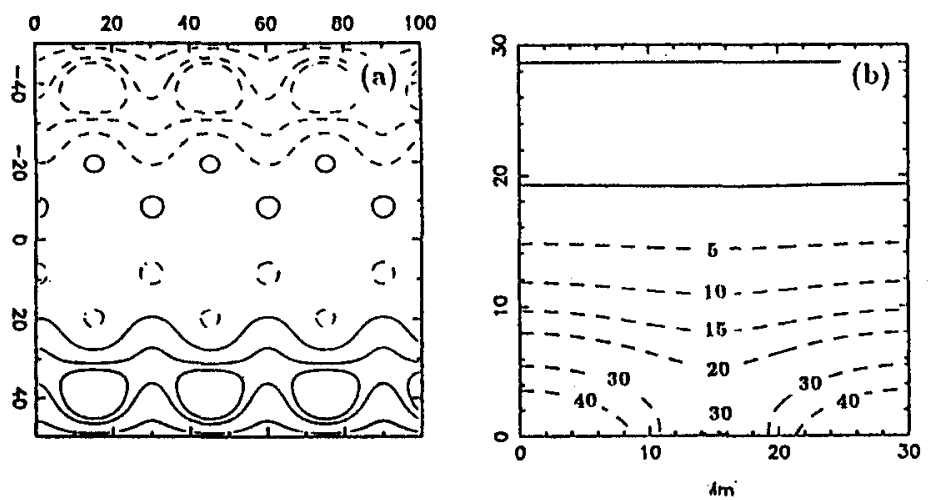

Figure 2. The vertical photosheric field is shown in (a). Full (resp. dashed) lines are given for $B_{z}>0$ (resp. $B_{z}<0$ ). The angle between the field and the prominence axis is shown in (b). 
bipolar pattern and the prominence stands above a magnetic corridor containing only small parasitic polarities (see Figure 2). Despite a high helicity, the combination of harmonics permits a large angle between the field and the prominence axis, as measured by the Hanlé effect (Bommier et al. 1994).

\section{The Natural Presence of Lateral Feet}

We compute the magnetic field in a 3-D cube, and we look for the dips everywhere in this region. The main prominence body and its underlying feet appear naturally. The feet reach periodically the photosphere, every $30 \mathrm{Mm}$, which is imposed as the mean size of supergranulation cells. A remarkable feature is the presence of dips aside from the prominence, forming a lateral dip structure. These features join the underlying feet and reach the photosphere, forming bald patches. Their presence is related to small parasitic magnetic polarities in the low field corridor around the inversion line. It is noteworthy that they can be guessed from the $2 \frac{1}{2}$-D OF topology (see Figure 1).

Then, assuming that the shape of the prominence is characterized by the presence of cold plasma contained in magnetic field lines at the dip locations, we only draw the portion of these which is likely to be filled with matter. We suppose that for each field line, the plasma can be observed on a few typical height scales above each dip. So we only draw the field lines at a given height of $1 \mathrm{Mm}$. The result is shown in Figure 3.

alplia $=0.09,11=i \mathrm{M} m$ SINISTRAL

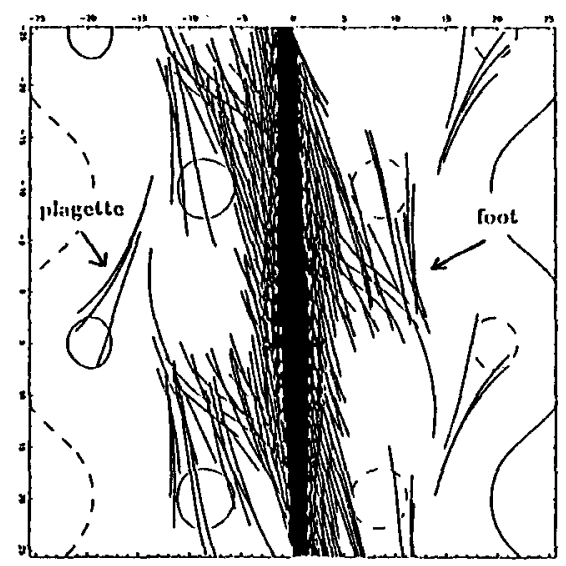

alplu $=-0.0 !, 11=1 \mathrm{Mm}$ DEXTILAL

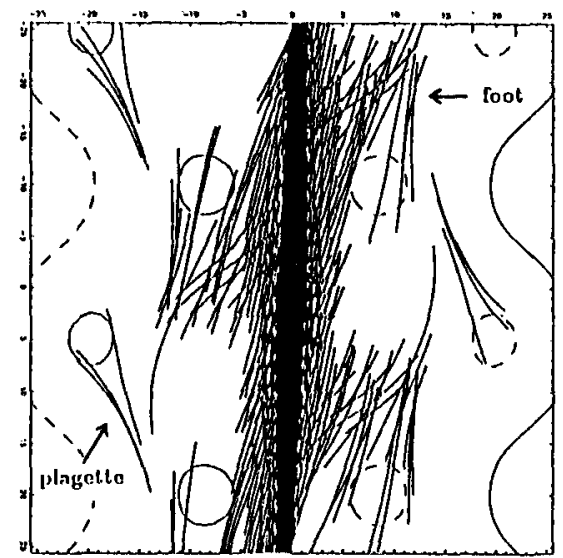

Figure 3. Field lines computed for the same parameters as Figure 2; they are only drawn at the dip locations at a given height of $1 \mathrm{Mm}$.

Viewed from above, the lateral feet structures are composed of some portions of field lines that look like what has been introduced as barbs by Martin et al. (1994). These structures match quite well those of observed regular filaments. They naturally appear periodically by pairs, alternating between both sides of the prominence. The orientation, shape and proximity of the represented field 
lines give the first impression that some field lines can cross the prominence, from one lateral foot to another one. This is not so. These two lateral features are in fact composed of different groups of field lines.

It is obvious in Figure 3 that a simple reversal of the sign of $\alpha$ changes the orientation of the lateral feet: A positive value of $\alpha$ gives a sinistral and left-bearing prominence, with overlying arcades having a right skew, and a righthanded helical field for the flux tube that supports the prominence. A negative $\alpha$ gives a dextral and right-bearing prominence, with overlying arcades having a left skew, and a left-handed helical field twist for the flux tube. This naturally links together the observed different patterns of chirality, in relation to the hemispheric helicity segregation (Martin and McAllister 1996, Rust and Kumar 1994, Pevtsov et al. 1995).

\section{Conclusions}

The magnetic configurations suited for prominence support we derived in the present paper are based on only a few hypotheses. We assume that the lfff approximation is valid to zeroth order and that combining a very small number of harmonics is sufficient to describe them. This simplicity provides very good agreement with many observational aspects of prominences and their environments, in particular the photospheric magnetic field, the measurements of the field in prominences made by the Hanlé effect, and the chirality pattern of the feet and overlying arcades. Further developments of the model are reported in Aulanier and Démoulin (1998). Finally, we note how fundamental the three-dimensional nature of the model was for the present study. It is obviously important for comparison with observations but, moreover, it emphasizes the importance of the OF configurations.

Acknowledgments. The authors thank L. van Driel-Gesztelyi for her help in the improvement of the text.

\section{References}

Aulanier, G. and Démoulin, P. 1998 A\&A, 329, 1125

Bommier, V., Landi Degl'Innocenti, E., Leroy, J.-L. and Sahal-Bréchot, S. 1994, Solar Phys., 154, 231

Démoulin, P., Priest, E.R. and Anzer, U. 1989, A\&A, 221, 326

Martin, S.F., Bilimoria, R. and Tracadas, P.W. 1994, in R. Rutten and C. Schrijvers (eds.), Solar Surface Magnetism, Kluwer, Dordrecht, Holland, p. 303

Martin, S.F. and McAllister, A.H. 1996, in Magnetodynamic Phenomena in the Solar Atmosphere, (eds.) Y. Uchida et al., Kluwer, Dordrecht, Holland, p. 497

Pevtsov, A.A., Canfield, R.C. and Metcalf, T.R. 1995, ApJ, 440, L109

Rust, D.M. and Kumar, A. 1994 Solar Phys., 155, 69

Titov, S., Priest, E.R. and Démoulin, P. 1993, A\&A, 276, 564 